Mens

Revue d'histoire intellectuelle et culturelle

mens

\title{
Les correspondances de Chauveau au Courrier des États-Unis, 1841-1849
}

\section{Lucie Robert}

Volume 19, numéro 1-2, automne 2018, printemps 2019

Les années 1840 : rupture ou réarticulation des possibles ?

URI : https://id.erudit.org/iderudit/1070073ar

DOI : https://doi.org/10.7202/1070073ar

Aller au sommaire du numéro

Éditeur(s)

Centre de recherche en civilisation canadienne-française

ISSN

1492-8647 (imprimé)

1927-9299 (numérique)

Découvrir la revue

Citer cet article

Robert, L. (2018). Les correspondances de Chauveau au Courrier des États-Unis, 1841-1849. Mens, 19(1-2), 165-183. https://doi.org/10.7202/1070073ar
Résumé de l'article

De 1841 à 1849, Pierre-Joseph-Olivier Chauveau (qui signe encore " P. C. », initiales de Pierre Chauveau) agit comme correspondant canadien du Courrier des États-Unis, que dirige alors à New York le journaliste français Théodore-Frédéric Gaillardet. Il y commente la vie politique canadienne et connaît ainsi une certaine notoriété puisque ses articles sont régulièrement reproduits et commentés dans la presse canadienne. Ces correspondances, parmi les premières du journalisme canadien, révèlent la manière dont le jeune Chauveau subsume l'angoisse suscitée par les rébellions ainsi que des traces d'un romantisme politique que sa carrière ultérieure saura éteindre, mais qui permet alors de mieux saisir la genèse de son roman Charles Guérin. 


\section{Les correspondances de Chauveau au Courrier des États-Unis, 1841-1849}

Lucie Robert

Université du Québec à Montréal et CRILCQ

\section{Résumé}

De 1841 à 1849, Pierre-Joseph-Olivier Chauveau (qui signe encore «P. C.», initiales de Pierre Chauveau) agit comme correspondant canadien du Courrier des États-Unis, que dirige alors à New York le journaliste français Théodore-Frédéric Gaillardet. Il y commente la vie politique canadienne et connaît ainsi une certaine notoriété puisque ses articles sont régulièrement reproduits et commentés dans la presse canadienne. Ces correspondances, parmi les premières du journalisme canadien, révèlent la manière dont le jeune Chauveau subsume l'angoisse suscitée par les rébellions ainsi que des traces d'un romantisme politique que sa carrière ultérieure saura éteindre, mais qui permet alors de mieux saisir la genèse de son roman Charles Guérin.

\section{Abstract}

From 1841 to 1849, Pierre-Joseph-Olivier Chauveau (who still signs "P. C.", initials of Pierre Chauveau) acts as the Canadian correspondent of the Courrier des États-Unis, then directed in New York by the French journalist Théodore-Frédéric Gaillardet. He comments on Canadian politics and is well known because his articles are regularly reproduced and commented on in the Canadian newspapers. These correspondences, among the first in Canadian journalism, reveal the way in which the young Chauveau subsumes the anguish provoked by the 
rebellions as well as traces of a political romanticism that his later career will extinguish, but which then makes it possible to better understand the genesis of his novel Charles Guérin.

Le jeudi 20 mai 1841 paraît dans Le Courrier des États-Unis une "Correspondance canadienne» signée des initiales «P. C.». C’est Étienne Parent qui aurait recommandé à Frédéric Gaillardet, le directeur du journal, la candidature de Pierre Chauveau qui, alors âgé de 21 ans, est encore un étudiant en droit qui termine sa formation par une année de cléricature chez George Okill Stuart, dont l'étude est une des plus réputées à Québec. En 1841, la signature «P. C.» est déjà connue des lecteurs du Canadien. Chauveau y a fait paraître sous ces initiales quelques poèmes et articles, parmi lesquels "L'insurrection", le 6 avril 1838, les "Adieux à Sir John Colborne", le 23 octobre 1839, et «L'Union des Canadas ou La fête des banquiers", le 5 avril 1841, trois poèmes qui révèlent un écrivain prometteur, mais aussi un jeune homme inquiet de l'avenir d'un pays et d'une communauté malmenés par les récents événements politiques qu'ont été les rébellions de 1837 et 1838 et l'Union des Canadas. J'insiste sur la signature, car signer «P. C.» n'est pas la même chose que signer «P.-J.-O. Chauveau» ou «Pierre-Joseph-Olivier Chauveau», ce que la même personne fera après 1850 . «P. C.» est un homme jeune, romantique, parfois effronté et certainement très ambitieux. P.-J.-O. Chauveau, comme il signera plus tard, à l'anglaise, est un homme politique d'âge mûr, conservateur et déjà ministre $^{1}$. Ces correspondances, parmi les premières du journalisme canadien, sont une des voies de sa venue à l'écriture. S'y exerce une

\footnotetext{
1 Sur ces premiers écrits de Chauveau et sur l'usage de la signature «P. C.», voir mes articles antérieurs : "Autour de "L'insurrection" du jeune Pierre Chauveau», dans Bernard Andrès et Marc André Bernier (dir.), Portrait des arts, des lettres et de l'éloquence au Québec (1760-1840), Paris, L'Harmattan; Québec, Presses de l'Université Laval, 2002, p. 327-345, ainsi que "Étranger à son temps et à lui-même." : l'écrivain et ses signatures ", Voix et images, no 88 (automne 2004), p. 31-46.
} 
plume habile et délurée, qui expérimente la description comme le dialogue et qui trouvera son principal débouché dans un des romans les plus intéressants du XIx ${ }^{e}$ siècle, Charles Guérin (1852). S’y révèle en même temps la manière dont le jeune Chauveau subsume l'angoisse suscitée par la tourmente politique qui a marqué son adolescence ainsi que les traces d'un romantisme politique que sa carrière ultérieure saura éteindre.

\section{Le Courrier des États-Unis sous Frédéric Gaillardet}

Journal de la communauté française de New York, Le Courrier des États-Unis, fondé en 1828, est alors publié sous une nouvelle direction, celle de Théodore-Frédéric Gaillardet (1808-1883), journaliste et écrivain français connu à Paris comme auteur dramatique et célèbre pour le procès qu'il avait intenté quelques années plus tôt (1832) à Alexandre Dumas père, prétendant être l'auteur d'une première version de La tour de Nesle, procès qu'il avait alors perdu. Projetant d'écrire un livre sur les États-Unis, à la manière de Tocqueville, Gaillardet débarque à la Nouvelle-Orléans en 1837, visite Cuba et le Texas, remonte le Mississippi jusqu'au Kentucky et publie ses reportages dans La Presse et le Journal des débats ${ }^{2}$. Au cours de ce voyage, il découvre avec étonnement la forte présence française sur le continent. De retour à New York, il achète Le Courrier des États-Unis, qui connaît alors d'importantes difficultés financières.

Le 31 décembre 1839, Gaillardet fait connaître son programme au lectorat du Courrier. Le journal continuera de publier des correspondances venues de France, mais il ajoutera désormais des correspondances en provenance d'autres endroits de l'Amérique. Il informera les Français de New York de la situation politique et

2 A. Krebs, "Gaillardet (Théodore-Frédéric) ", Dictionnaire de biographie française, t. Xv, Paris, Librairie Letouzey et Ané, 1982, p. 108. Le livre annoncé paraît après la mort de l'auteur en 1883, sous le titre L'aristocratie en Amérique, Paris, E. Dentu, éditeur, Librairie de la Société des Gens de Lettres. Sur la page de titre, la signature désigne Gaillardet comme «ancien rédacteur en chef du Courrier des Etats-Unis». 
culturelle de la France, soutiendra la langue et les mœurs françaises sur le continent américain, intégrera au lectorat du Courrier les Canadiens, les Créoles de la Louisiane et ceux des Caraïbes, fera connaître la francophonie du continent américain aux Français de New York. L'objectif de Gaillardet est «de relier et d'unir entre elles, par la pensée, chacune des communautés Françaises de l'Amérique $[\ldots]^{3} »$. Aussi, et à la différence de ses prédécesseurs, Gaillardet consacre la majorité des premières pages de son journal à l'actualité étatsunienne et il entreprend de recruter des correspondants de toutes les régions francophones d'Amérique. Dans les années qui suivent, il va aussi voyager dans cette Amérique, dans cette communauté française, pour vendre son journal. On le retrouve à l'hiver 1842, à Cuba, au printemps à la Louisiane, puis à Pittsburgh et dans la vallée du Mississippi et de l'Ohio. À l'été 1843, il est reçu au Canada, qu'il visite depuis Québec jusqu’à Kingston, avant de poursuivre, au printemps 1844, vers l'ouest et le sud des ÉtatsUnis. Gaillardet donne ainsi un solide coup de barre au Courrier des États-Unis, qui battait de l'aile quand il en a pris possession. À l'automne 1839, le journal comptait 600 abonnés et, au printemps 1840 , il en compte $2200^{4}$. Le journal est devenu trihebdomadaire, paraissant le mardi, le jeudi et le samedi, et il s'est doté d'un supplément intitulé La Semaine littéraire. Gaillardet dirige le journal jusqu'en 1848, quand, à la faveur de la nouvelle république, il croit pouvoir reprendre sa carrière en France. Il vend alors le journal à Paul Arpin, un journaliste de la Nouvelle-Orléans, mais il reste un correspondant fidèle du journal.

3 Le Courrier des États-Unis, 16 janvier 1840. Toutes les citations respectent l'orthographe et la syntaxe de l'époque, qui peut varier selon les locuteurs. Les renseignements sur Le Courrier des États-Unis proviennent d'Anthony GrolleauFricard, Le Courrier des États-Unis : entre France, Etats-Unis et Canada (18281851), thèse de doctorat (histoire), Paris-I Panthéon-Sorbonne, 2009 et d'Yvan Lamonde, "Le Bas-Canada et Le Courrier des États-Unis de New York (18281840)", Les Cahiers des Dix, no 56 (2002), p. 217-233.

4 Grolleau-Fricard, Le Courrier des États-Unis : entre France, États-Unis et Canada (1828-1851), p. 13. 
Lidée d'établir un réseau de correspondants n'est pas propre au Courrier des États-Unis. Depuis longtemps, les journaux canadiens publient ces articles qui informent les lecteurs de la vie et de la politique européenne 5 . La pratique la plus courante est celle du repiquage, avec ou sans autorisation, d'articles issus de la presse européenne. L'état de la circulation de la poste ne permet pas de rendre compte de l'événement au moment où il se produit. Il faut plusieurs jours, voire quelques semaines, avant que la malle-poste ne parvienne de Paris à New York, puis de New York à Montréal, par exemple, ayant le plus souvent transité par Londres et Liverpool. Même sur le continent américain, le bateau met plusieurs jours à descendre la rivière Hudson entre Montréal et New York. La correspondance cherche à combler cette distance: elle a pour mission d'informer, mais elle a aussi celle de commenter l'événement après coup. Elle se présente généralement sous la forme d'une lettre datée et signée, adressée au directeur du journal, et elle s’apparente souvent au reportage parlementaire bien qu'elle soit aussi déjà une forme de feuilleton composé de longs essais multiformes publiés à intervalles à peu près réguliers, tirant profit d'une certaine liberté de ton propre à la forme-lettre ${ }^{6}$. La plupart des correspondances des années 1840 ne sont pas signées, mais celles qui le sont engagent, plus que les autres, la fidélité du lectorat par l'unité de ton et de point de vue que cette signature impose.

En 1840, les correspondances ne sont pas nouvelles non plus au Courrier des États-Unis. En l'absence de la fonction de

5 Pierre-Louis Lapointe, «La nouvelle européenne et la presse québécoise d'expression française (1866-1871)", Revue d'histoire de l'Amérique française, vol. XXVIII, $\mathrm{n}^{\circ} 4$ (mars 1975), p. 517-537.

6 Guillaume Pinson (dir.), «La lettre et la presse: poétique de l'intime et culture médiatique», Médias 19, 2012 [En ligne]. Voir, en particulier, l'article d'Éloïse Pontbriand, "Chroniques parlementaires, chroniques alimentaires: le cas des "Lettres de Bordeaux" d'Émile Zola», et celui de José-Luis Diaz, "Presse et épistolaire au XIx ${ }^{\mathrm{e}}$ siècle: la scansion des Lettres parisiennes de Mme de Girardin (1836-1843)", Médias 19, 2012, [En ligne], [http://www.medias19.org/index. php?id=275] (5 octobre 2018). 
journaliste-reporter, profession qui n'existe pas à cette époque, l'information est soit empruntée aux autres journaux (couper-coller, repiquer, avec ou sans entente légale ${ }^{7}$ ), soit transmise par les correspondants, dont les lettres forment depuis le début du journal le coeur ou le noyau du système d'information. Avec l'arrivée de Gaillardet, soutient Anthony Grolleau-Fricard, "les correspondances occupe[ro]nt une place centrale dans Le Courrier des ÉtatsUnis $^{8}$ ». Dans sa thèse, celui-ci en a compté 70 en 1840 et 113 en 1841. Il note: «Le nombre de correspondances oscille d'une année sur l'autre. Il atteint son plus bas en 1847 avec 61 correspondances ${ }^{9}$." Le nombre de ces correspondances augmente encore sous la direction de Paul Arpin et, en 1850, on en compte 134. Le nombre de rédacteurs de correspondances (les correspondants) augmente lui aussi. Il est de 12 en 1841, de 15 en 1847, de 24 en 1844, puis à la baisse entre 1848 et 1850 . Ceux-ci sont "plus ou moins réguliers, [ils] écrivent leurs chroniques depuis Paris ou depuis d'autres lieux du continent américain [et] viennent apporter leurs témoignages sur la vie locale, mais aussi sur des événements importants auxquels ils assistent ou encore participent ${ }^{10}{ }^{\prime}$. En 1840, seulement trois des seize correspondants écrivent plus de cinq articles. En 1841, la proportion sera de la moitié (soit 7) avant de retomber au quart en 1845.

Le Courrier des États-Unis est diffusé au Bas-Canada depuis sa fondation. On connait au moins deux de ses agents en 1833: Hector Bossange à Montréal et Samuel Neilson à Québec. Entre 1831 et 1837, Le Courrier des États-Unis est le journal américain le plus fréquemment cité dans la presse canadienne, et c'est aussi

7 Will Slauter, "Le paragraphe mobile: circulation et transformation des informations dans le monde atlantique du XviII ${ }^{\mathrm{e}}$ siècle", Annales. Histoire, Sciences sociales, vol. XLVII (2012), p. 363-389; Ryan Cordell, «Reprinting, Circulation, and the Network Author in Antebellum Newpapers ", American Literary History, vol. XXVII, ${ }^{\circ} 3$ (2015), p. 417-445.

8 Grolleau-Fricard, Le Courrier des États-Unis : entre France, États-Unis et Canada (1828-1851), p. 156.

9 Ibid.

${ }^{10}$ Ibid., p. 159. 
visiblement lui qui fournit celle-ci en informations étatsuniennes. Il est difficile cependant de juger de son importance dans la transmission des nouvelles européennes puisque les articles repiqués citent plutôt la source originale que le véhicule de transmission. En revanche, l'actualité bas-canadienne est peu relayée au Courrier des États-Unis, comme l'a montré Yvan Lamonde ${ }^{11}$, qui note que, jusqu'en 1840, elle y figure d'abord pour des événements de nature exceptionnelle (dont les deux rébellions) et, surtout, qu'elle y figure par des articles empruntés à la presse loyaliste, par exemple L'Ami du peuple, de l'ordre et des lois, journal sulpicien, ou, encore plus souvent, de la presse anglophone, qui peint les Canadiens comme des malfaiteurs, qui échappent à l'autorité britannique. Sous la direction de Gaillardet, lui-même plutôt antibritannique, l'actualité canadienne prend de l'importance en même temps que les journaux de langue française auxquels il emprunte l'information. L'actualité canadienne occupera jusqu'à $13,79 \%$ des unes ${ }^{12}$ du journal sous son directorat. Après son départ, la situation revient à ce qu'elle était avant son arrivée, et il n'y a presque plus de premières pages consacrées au Canada après 1850.

\section{Pierre Chauveau au Courrier des États-Unis}

Le 14 août 1840, Chauveau avait publié anonymement - quoique l'anonymat à Québec et dans Le Canadien soit une chose bien relative -, une série de neuf questions satiriques portant sur le projet d'union des Canadas ${ }^{13}$, questions dont il reconnaîtra ultérieurement la paternité dans une note de son ouvrage sur François-Xavier Garneau ${ }^{14}$. Dans ces questions, qui sont en réalité des propositions

${ }^{11}$ Lamonde, "Le Bas-Canada et Le Courrier des États-Unis de New York (1828-1840)».

${ }^{12}$ Grolleau-Fricard, Le Courrier des États-Unis : entre France, États-Unis et Canada (1828-1851), p. 489.

${ }^{13}$ L'article paraît sous la forme d'une lettre à l'éditeur, surtitrée "Aide-toi! Et le ciel t'aidera!», dans Le Canadien, 14 août 1840, p. 1.

${ }^{14}$ Pierre-Joseph-Olivier Chauveau, François-Xavier Garneau, sa vie et ses auvres, 
déguisées puisqu'elles sont toutes formulées sous la forme «Au lieu de [...] ne vaudrait-il pas mieux [...]?», Chauveau recommande de préparer les élections avec soin, de continuer à rédiger des pétitions destinées au gouvernement impérial pour réclamer une représentation juste et proportionnelle aux deux provinces et d'exiger des juges et des magistrats compétents. Comme Étienne Parent, il demande que les Canadiens réfutent le discours francophobe des Britanniques:

[...] ne vaudrait-il pas mieux nous énorgueillir des vertus que nous avons $[\ldots]$, moins mépriser tout ce que font nos compatriotes, tenir un peu plus à notre langue, ne pas rougir de la parler devant ceux qui en parlent une autre, et surtout ne pas nous afficher exclusivement en anglais comme on le voit sur toutes nos enseignes ${ }^{15}$ ?

Puis il dégage un programme fondé sur l'association financière qui permettrait «d'exploiter la vaste récolte commerciale et industrielle» du pays, sur le développement d'un enseignement secondaire destiné à « orner notre pays de bons et braves marchands, gros et gras, et d'industriels actifs, riches et éclairés" et sur la création d'une association visant à " procurer des secours à ceux de nos jeunes gens qui désirent s'établir dans les townships de l'une ou de l'autre province ${ }^{16}{ }$. Ainsi, ce n'est pas la question coloniale qui prime alors aux yeux de Chauveau, mais bien le développement économique et l'occupation du territoire. Ce sont là les idées qu'il défendra dans les pages du Courrier des Etats-Unis.

L'Union des Canadas est proclamée six mois plus tard, le 10 février 1841. Le 2 avril, paraît à la une du Canadien l' "Adresse aux électeurs du comté de Terrebonne» de Louis-Hippolyte LaFontaine.

Montréal, Beauchemin et Valois, 1883, p. LII, note. L'exemplaire mis en ligne dans la collection numérique de BAnQ porte une indication manuscrite qui attribue l'article à P. Chauveau. Voir [En ligne], [http://numerique.banq.qc.ca/ patrimoine/details/52327/3455229] (5 octobre 2018).

15 Le Canadien, 14 août 1840 , p. 1.

${ }^{16}$ Ibid. 
Chauveau se rappellera ces années «qui virent s'accomplir l'Union législative du Haut et du Bas-Canada [comme] une époque cruelle dans notre histoire", une époque "qui fut difficile à traverser pour les cœurs généreux, pour les esprits imbus d'idées patriotiques", une époque dont il écrira qu' elle fut celle d'une "seconde conquête ${ }^{17}$ ". Il mettra quelque temps à accepter l'Union des Canadas et à se rallier à l'«Adresse aux électeurs du comté de Terrebonne» de LaFontaine. Entre-temps, il fait partie de cette cohorte de jeunes gens qui, écrira-t-il, "cherchait les moyens de conserver ou plutôt de raviver le patriotisme ${ }^{18} »$. Ses correspondances au Courrier des ÉtatsUnis témoignent de cet effort. Laurent-Olivier David a-t-il bonne mémoire lorsqu'il rappelle que " [1] es Canadiens-Français saluèrent avec joie des accents patriotiques qui les transportaient, et virent dans le jeune Chauveau un homme de l'avenir, un soldat brillant de la cause nationale ${ }^{19}$ "?

Le 20 mai 1841 paraît ainsi la première "Correspondance canadienne» de «P. C.» dans Le Courrier des États-Unis. Cinq autres paraîtront la même année, le plus souvent à la une. L'auteur rédige une chronique parlementaire où il ne cache pas ses opinions, commentant avec un humour souvent caustique les tribulations des divers gouverneurs, lord Sydenham (Poulett Thompson), sir Charles Bagot, lord Metcalfe, et dénonçant le rôle des banquiers dans la formation de l'Union. Il prend le temps de retracer l'histoire récente du Canada-Uni, remontant au mouvement patriote et aux résolutions Russell (donc en 1834), et d'expliquer la configuration des partis politiques en présence. Il s'adresse explicitement aux Français des États-Unis, à ce "vous récemment venu de l'arrière-pays de France» (29 juin 1841), et il conserve un ton ironique pour décrire le «Breton d'Amérique, qui se croit un Jupiter exilé et qui lance

${ }^{17}$ Chauveau, François-Xavier Garneau, sa vie et ses æuvres, p. v.

${ }^{18}$ Ibid., p. viII.

${ }^{19}$ Laurent-Olivier David, L'Honorable P-J.-O. Chauveau, Montréal, Typographie Geo.-É. Desbarats, 1872, p. 9. 
l'injure la plus triviale, comme la seule foudre qui lui reste» (29 juin 1841). Le gouvernement est décrit comme un théâtre et l'activité politique comme une comédie: "Quoiqu'il en soit, au moment où je vous écris, le rideau doit être tiré et les acteurs en scène. Nous ne connaissons que ceux du premier acte» (29 juin 1841). Il énonce son programme: "J'espère, à l'avenir, selon les sujets que me jetteront les événemens, donner de toutes les phases des affaires canadiennes des esquisses consciencieuses pour lesquelles je réclame votre indulgence et surtout celle de mes compatriotes» (29 juin 1841), ajoutant aussitôt, mais de manière plus ponctuelle: "Espérant avoir à vous signaler quelque démarche importante de notre corps législatif, j'ai retardé pendant plusieurs jours l'envoi de ma correspondance mensuelle; mais, voyant que j'attendais en pure perte, j'ai pris mon parti. Jusqu'à présent, la chambre n’a rien fait» (29 juin 1841). Ce motif du «rien à dire» revient plusieurs fois au cours des correspondances. Le 28 septembre 1844, par exemple, Chauveau écrit: "Je n'ai pas besoin de m'excuser auprès de vous de mon long silence." C'est que, ajoute-t-il, il n'avait rien à dire que répéter les chroniques précédentes sur les querelles entre Denis-Benjamin Viger et la presse libérale.

En 1842 paraissent trois correspondances. Elles sont rédigées de la même manière, poursuivant l'énoncé des «misérables vexations» ( $1^{\text {er }}$ avril 1842), ce qu'il appellera aussi la «longue trame des sottes iniquités» (16 décembre 1845), que le gouvernement britannique impose aux Canadiens, tout en expliquant au passage à ses lecteurs ce qu'est une «bordée» de neige et ce qu'est la "poudrerie». Il prend le temps de cartographier la presse canadienne, décrivant les journaux selon leurs orientations politiques. Ainsi, La Gazette de Québec

représente le juste milieu, dont M. Nelson s'est trouvé le chef toutes les fois qu'il n'a pas été à l'extrême-droite ou à l'extrême-gauche. [...] Le Fantasque, dont le genre est la satire, et qui l'exerce toujours contre le parti du plus fort [...], parle à peu près le langage de l'extrême-gauche [et] le Canadien peut 
être considéré comme représentant le centre-gauche. Quant au Herald, il fait partie des journaux anti-français. (8 octobre 1842)

Il y a donc six chroniques en 1841 , trois en 1842, deux en 1843 , quatre autres en 1844. Plus de la moitié des chroniques sont ainsi publiées dans les trois premières années. Elles sont reproduites et commentées dans les journaux canadiens : parfois dans Le Canadien, régulièrement dans Le Castor et même dans L'Avenir. L'Aurore des Canadas les commente aussi, mais sous un autre mode, plus polémique, voire plus pervers, ne les citant pas vraiment ou les citant de travers. La dixième correspondance, datée du $1^{\text {er }}$ juin 1843, est titrée «De l'état présent et de l'avenir du Canada». C'est la première à porter un titre. La suivante sera intitulée «Situation présente du Canada». Ces deux chroniques sont les plus fréquemment reproduites dans les journaux canadiens, qui n'ont pourtant pas négligé les copier-coller depuis le début et qui n’ont pas négligé non plus de déployer des polémiques, au point où Chauveau, signant toujours «P. C.», prend parfois la peine de répliquer, mais seulement dans les journaux canadiens ${ }^{20}$. Car les correspondances ont du succès, du moins du côté canadien de la frontière. On y sent, en effet, monter dramatiquement la tension dans le parlement canadien, où les conflits sont de plus en plus manifestes. À propos de la discussion relative au siège du gouvernement, Chauveau parlera d' «une crise des plus délirantes», créée par "des aristocrates au petit-pied» (23 novembre 1843). Le 28 septembre 1844, il laisse entrevoir la possibilité d'une campagne électorale: «[I]l faudra toujours bien, dans les premiers mois de 1845, se trouver en face du pays, en face d'une élection générale».

Pourtant, le 30 septembre 1844, soit deux jours après avoir annoncé cette hypothétique campagne électorale, mais, on l'a vu,

${ }^{20}$ Antoine Gérin-Lajoie reproduit plusieurs extraits de chroniques sur l'Union, datées de décembre 1841, de juin 1843, de novembre 1843, de décembre 1843 et d'avril 1844. Voir Dix ans au Canada, de 1840 à 1850: histoire de l'établissement du gouvernement responsable, Québec, Typographie de L. J. Demers \& Frères, 1888. 
pour "les premiers mois de 1845 ", soit bien plus tard, Chauveau publie son adresse " [a]ux libres et indépendants électeurs du comté de Québec». "Inaugurant sa campagne, le $1^{\text {er }}$ octobre, devant l'église Saint-Roch, à Québec, [il] dévoile un programme en trois points: le gouvernement responsable, le progrès de l'instruction et celui de l'industrie. Il résume ses projets en une phrase: "Le plus grand bien du plus grand nombre ${ }^{21 "}$." Le 23 octobre, Le Canadien publie le résultat du scrutin. Chauveau l'emporte, ayant obtenu 1543 voix contre son adversaire, John Neilson, qui doit se contenter de 458 voix. Il l'emporte dans cinq des huit quartiers (SaintRoch, Haute-Ville, Beauport, Sainte-Foy, L’Ancienne-Lorette); les résultats sont égaux dans Saint-Dunstan, alors que Neilson l'emporte dans deux quartiers (Saint-Ambroise et Charlesbourg). Le 25 octobre paraissent ses remerciements "[a]ux libres et indépendants électeurs du comté de Québec». Chauveau restera député du comté de Québec jusqu'en juillet 1855 et, au Parlement, il appuiera généralement les réformistes de Louis-Hippolyte LaFontaine et de Robert Baldwin. C'est en français qu'il prononce son premier discours. Arthur Beauchesne écrit: "Le jeune Pierre-Joseph-Olivier Chauveau se leva alors pour dire qu'il devait se hâter de parler tandis que la présidence était occupée par une personne capable de comprendre sa langue maternelle ${ }^{22}$.»

La quinzième chronique, datée du 23 novembre (et publiée le 3 décembre), celle qui suit ces élections, revient vaguement sur l'événement. Chauveau abuse sans doute de la crédulité de ses lecteurs en parlant de ces "élections provinciales, que personne ne prévoyait comme aussi prochaines, lors de l'envoi de ma dernière lettre, mais qui étaient déjà décrétées" alors que, au moment de la publication de son texte, il préparait déjà sa campagne électorale. Il

\footnotetext{
${ }^{21}$ Pierre Poulin et Jean Hamelin, "Chauveau, Pierre-Joseph-Olivier», Dictionnaire biographique du Canada, version révisée en ligne, [http://www.biographi.ca/fr/ bio/chauveau_pierre_joseph_olivier_11F.html] (5 octobre 2018).

${ }^{22}$ Arthur Beauchesne, "Pierre Chauveau ", La Revue moderne, vol. III, n 3 (15 janvier 1922), p. 24.
} 
ajoute encore cependant: "L'union des Canadas est une monstruosité». Il faudra six mois avant que ne paraisse la chronique suivante. Forcément, une fois membre de ce Parlement qu'il n'a eu de cesse de dénoncer, participant d'un gouvernement qui tente tant bien que mal de rétablir un semblant de paix sociale, "P. C." est tenu à une certaine réserve. Les correspondances qui paraissent après son élection seront donc toujours des chroniques parlementaires, et l'auteur évite la discussion des projets de loi en débats, commentant davantage la fracture qui, chez les Canadiens, opposera le clan PapineauViger au parti gouvernemental.

Suivront quand même, en 1845, trois belles chroniques portant sur les incendies de Québec: celle du $1^{\text {er }}$ juin, visiblement écrite dans l'urgence, raconte l'incendie du quartier Saint-Roch et appelle à l'aide internationale. Elle est précédée d'une note de la rédaction qui annonce une souscription pour les habitants de Québec et elle se termine par une autre note de la rédaction expliquant que le journal met à la disposition de qui veut le voir, un plan de la ville de Québec "sur lequel est indiquée toute la partie qui a été incendiée». La correspondance du 30 juin décrit l'incendie du faubourg Saint-Jean à la Haute-Ville. Le Courrier des États-Unis commence par reproduire un article du Canadien, qu'il fait suivre de la lettre de Chauveau. Le 15 octobre, nous avons droit au bilan. L'article ne paraît pas à la une. Chauveau rappelle l'ampleur de la catastrophe et insiste sur la générosité de l'Angleterre (il aimerait que la France en fasse autant). Suit, de manière un peu inattendue, l'annonce de la parution de l'Histoire du Canada de François-Xavier Garneau, dont il fait un compte rendu assez rapide: «[L]'Histoire du Canada ne doit pas être indifférente à vos hommes d'État, qui paraissent prendre à cœur la colonisation de l'Algérie et de la Guyane française. Si M. Garneau se maintient, dans ses quatre volumes, à la hauteur de son début, il aura écrit la première histoire complète de son pays ». Et il ne peut s'empêcher de noter: «M. Garneau a cependant choisi un mauvais moment pour lancer son livre. Les sinistres de cet été ont plongé la population entière dans une stupeur très peu 
favorable à l'appréciation d'une œuvre semblable.» La rédaction du Courrier des États-Unis répond:

Notre correspondant s'est chargé de réparer le silence involontaire que nous avons gardé, jusqu'ici, sur cet ouvrage dont nous n'avons pas parlé encore, parce que, faute de tems, nous n'avons pas pu le lire avec l'attention nécessaire pour lui rendre toute la justice dont il est digne. Mais nous le connaissons déjà assez pour ne pas hésiter à nous associer aux éloges que lui adresse un homme mieux placé que nous pour en apprécier tous les mérites.

Puis Chauveau poursuit sa chronique en revenant sur les affaires parlementaires à propos de "[d]eux événemens qui, dans tout autre temps, auraient excité la plus vive sensation, [mais qui] sont passés presqu'inaperçus", soit l'élection de Denis-Benjamin Viger, dans le comté de Trois-Rivières, et celle d'André Taschereau, dans le comté de Dorchester, l'un et l'autre servant de paravent au "grand événement qui domine aujourd'hui toutes les préoccupations de nos hommes politiques. Après huit ans d'absence, Louis-Joseph Papineau est de retour dans son pays». De ce dernier événement cependant, Chauveau ne parle pas et l'on peut constater avec lui que «[c]e peu de mots donne beaucoup à penser ». On sent venir l'heure du bilan: "De 1837 à 1845, il s'est passé bien des choses ", note-t-il le 23 octobre 1845, sans préciser davantage.

En 1846, il ne paraît aucune correspondance de «P. C.». C'est cette année-là que les premiers chapitres du roman Charles Guérin paraissent anonymement dans l'Album littéraire et musical de la Revue canadienne. C'est aussi dans ces années que le nombre de correspondances publiées par le journal commence à diminuer. Il y aura encore une correspondance le 29 juin 1847 (datée du 21 juin) et le 18 mars 1848, dans laquelle Le Courrier des États-Unis annonce la formation du nouveau gouvernement Baldwin-LaFontaine. Le même jour paraît l'annonce de la révolution de février 1848, à Paris. Aucun autre article ne paraîtra sur le Bas-Canada jusqu'au départ de Frédéric Gaillardet. L'avant-dernière lettre de Chauveau, publiée le 28 décembre 1848, soit dix-huit mois après la précédente, sous 
la nouvelle direction de Paul Arpin, est précédée d'une note de la rédaction: "L'arrivée moins fréquente des steamers va nous permettre de suivre de nouveau avec attention le développement de faits que les événements Européens avaient nécessairement rejeté quelque peu dans l'ombre.» Dans sa lettre, Chauveau commence par s'adresser à Arpin: "Je n'ignore pas que votre feuille, pleine du récit des événemens qui bouleversent à l'heure présente le monde européen, ne peut abandonner qu'un très petit espace à nos démêlés coloniaux, aussi ne serai-je pas moins sobre que par le passé de détails et de discussions.»

La dernière correspondance est datée du 25 juillet 1849 et fait état des partis politiques au moment où se réunit l'Assemblée législative à Kingston. On constate que les enjeux ont changé: pour Chauveau, l'opposition principale est désormais entre «le parti de la connexion britannique, et celui de l'annexion aux États-Unis». En novembre, il refusera de signer le manifeste antiannexionniste: « $\mathrm{Si}$ les annexionnistes allaient avoir le dessus, nous nous serions placés dans une situation compromettante ${ }^{23}$ ", aurait-il déclaré à son collègue Louis-Joseph Massue. Néanmoins, sa position est claire et elle s'oppose à celle du Courrier des États-Unis, dont le nouveau directeur, Paul Arpin, favorise l'annexion du Bas-Canada aux États-Unis.

\section{Opter pour la carrière politique}

Peut-être les divergences politiques entre le républicain Arpin et le désormais conservateur Chauveau ont-elle été la cause d'une mésentente qui aurait conduit Chauveau à interrompre ses correspondances. Peut-être s'agit-il simplement du fait qu'Arpin ne s'intéressait pas à la cause des Canadiens, mais seulement aux effets que les désordres au Canada pourraient avoir sur les États-Unis, comme ceux qu’aurait entraînés un changement de régime, par exemple. Toutefois, il est difficile de croire qu'un correspondant repoussé par

${ }^{23}$ Cité par Pierre Poulin et Jean Hamelin, "Chauveau, Pierre-Joseph-Olivier». 
la direction du journal pour désaccord politique se soit arrêté aux bureaux du Courrier des États-Unis, en mai 1850, pour le simple plaisir de rencontrer en personne son directeur, dont il écrira:

M. Arpin fut des trois [personnes que je rencontrai] celui que me plut d'avantage; pour la franchise, et la bonhomie de ses manières. C'est un grand et gros homme noir un peu moins qu'un nègre: une barbe à la Eugène Sue; et des yeux noirs de la plus grande vivacité. Manières cordiales, et physionomie ouverte, presque comme un Canadien: quelque chose dans le genre d'Étienne Parent quoiqu'un peu plus verni ${ }^{24}$.

Pour expliquer ce silence, il faut sans doute plutôt revenir au fil des événements. En effet, en janvier 1848, Chauveau a été réélu, sans opposition, député du comté de Québec à la troisième législature du Canada-Uni. Le 29 mai 1849, soit deux mois avant sa dernière lettre, il a déposé à l'Assemblée législative son rapport sur l'émigration aux États-Unis. Il était président du comité spécial chargé de faire enquête. Enfin, en novembre 1851, il est nommé solliciteur général du Bas-Canada, dans le gouvernement Hincks-Morin. Il aura donc montré la loyauté et l'assiduité nécessaires pour obtenir ces fonctions, lesquelles sont difficilement compatibles avec la correspondance parlementaire. La raison politique se conjugue ici aux raisons financières et personnelles, car Chauveau n'est pas riche et il a une famille à nourrir. La lettre du 29 mai 1849 est aussi, à ma connaissance, le dernier texte que Chauveau signera «P. C.». En 1850, la trajectoire de Chauveau, à l'enseigne de cette double figure caractéristique du romantisme français, à la fois homme d'État et écrivain, sur le modèle des carrières de Lamartine et de Victor Hugo, est devenue impossible. Là où Lamartine et Hugo ont finalement opté pour la littérature, Chauveau opte pour la politique. Un autre personnage apparaît, celui de Pierre-Joseph-Olivier Chauveau, destiné à une longue

${ }^{24}$ Pierre-Joseph-Olivier Chauveau, Sept jours aux États-Unis, 1850, Québec, Éditions Nota bene, 2003, p. 117. 
carrière, à une plus longue carrière que celle de "P. C.", il faut bien l'admettre.

Ainsi, entre le 20 mai 1841 et le 25 juillet 1849, Chauveau aura publié 25 correspondances au Courrier des États-Unis. La moitié de ces correspondances ont paru au cours des trois premières années. Il serait excessif de prétendre que cette collaboration fut régulière jusqu'en 1850 . Entre 1844, date de la première élection de Chauveau, et 1850 , date de sa nomination au poste de solliciteur général du Bas-Canada, la plupart des correspondances portent sur des sujets autres que la vie parlementaire. Elles présentent un caractère moins critique et surtout moins personnel que les précédentes. Au cours de ces années, il aura été toutefois le seul correspondant canadien du journal et, selon Anthony Grolleau-Fricard, il aura été le plus régulier des correspondants. Après lui, il n’y en aura pas d'autres. Le Courrier des Etats-Unis est alors un des rares, sinon le seul journal non canadien à diffuser une information en français de première main sur le Bas-Canada et il le fait sous la plume unique de Chauveau. Cependant, on n'exagérera pas l'influence qu'ont pu exercer ces correspondances sur le lectorat européen, considérant le fait qu'aucune bibliothèque européenne ne possède de collection complète du Courrier même si, en principe, plusieurs y étaient abonnées. Néanmoins, ces écrits ont en leur temps fait contrepoids à l'information véhiculée par les journaux britanniques à un moment crucial de l'histoire du Bas-Canada.

Quelle que soit la manière de comparer, force est également de constater que les correspondances de Chauveau sont les premières de ce type ${ }^{25}$. Il y a là l'inauguration d'une pratique, qui emprunte,

${ }^{25}$ Sur la correspondance comme genre littéraire à cette époque, on lira Maurice Lemire et Denis Saint-Jacques (dir.), La vie littéraire au Québec, t. III : Un peuple sans histoire ni littérature, Québec, Presses de l'Université Laval, 1996, p. 331339. On distinguera ici la "correspondance» de la "chronique»: le correspondant n'est pas l'échotier, le causeur ou le flâneur que décrit Marie-Ève Thérenty dans La littérature au quotidien: poétiques journalistiques au XIX' siècle, Paris, Seuil, 2007. 
bien entendu, aux modèles étrangers ${ }^{26}$, mais qui les naturalise rapidement. Rien de tel avant, ni dans les journaux canadiens, qui ne comptent guère de correspondants étrangers sur une base régulière, ni dans les journaux étrangers, qui ne comptent guère de correspondants canadiens sauf peut-être, pour un bref moment, en 18311832, quand Philippe-Ignace-François Aubert de Gaspé repique des articles de La Minerve au profit de L'Abeille de la NouvelleOrléans et, en 1834, quand Napoléon Aubin envoie onze lettres de la Nouvelle-Orléans à La Minerve ${ }^{27}$. Chauveau a précisément fait ses classes avec Aubin, qu'il a fréquenté à Québec, et il a sans doute conscience du fait que ses correspondances l'inscrivent au cœur d'un système qui est déjà de l'ordre de la «mondialisation médiatique ${ }^{28}$ ", selon l'expression de Guillaume Pinson, où l'information circule dans un faisceau d'échanges croisés dans l'espace de la francophonie nord-américaine, mais aussi dans un mouvement transatlantique qui ouvre vers la France et la Grande-Bretagne. Chauveau, qui fréquente la bibliothèque de la Chambre d'assemblée depuis l'âge de treize ans ${ }^{29}$ et qui témoigne tout au long de sa carrière d'une solide culture, littéraire et politique, la première surtout restant exceptionnelle pour l'époque, a certainement tiré un immense profit de sa lecture des journaux, et son projet d'écrire un Essai sur la littérature française depuis 1789, ouvrage qui ne parut jamais cependant, montre cette ambitieuse ouverture à la France ${ }^{30}$.

${ }^{26}$ Marie-Ève Thérenty et Alain Vaillant, Presse, nation et mondialisation au XIX siècle, Paris, Nouveau Monde éditions, 2019, 512 p.

${ }^{27}$ Lamonde, «Le Bas-Canada et Le Courrier des États-Unis de New York (18281840)», p. 221.

${ }^{28}$ Guillaume Pinson, La culture médiatique francophone en Europe et en Amérique du Nord: de 1760 à la veille de la Seconde Guerre mondiale, Québec, Presses de l'Université Laval, 2016, p. 72.

${ }^{29}$ C'est, du moins, ce que laisse entendre Étienne Parent, dans le compte rendu des exercices philosophiques et littéraires, menés par les élèves de la classe de Chauveau au Petit Séminaire de Québec, qu'il publie dans Le Canadien, 16 août 1833, p. 2-3.

${ }^{30}$ Sur ce projet d'ouvrage, voir mon étude, "Germaine de Staël, aux origines de l'histoire littéraire au Québec», dans Martin Doré et Doris Jakubec (dir.), Deux 
De ce point de vue, les correspondances de Chauveau au Courrier des États-Unis forment les assises de sa carrière politique. C'est, en effet, de cette reconnaissance extérieure au pays que Chauveau, malgré son jeune âge, tire sa notoriété. Car Le Courrier des États-Unis conserve un important réseau de distribution sur l'ensemble du continent nord-américain, ce qui permet à la plume de Chauveau de se faire connaître dans un espace francophone élargi. De même, Le Courrier est bien distribué au Canada, où il est lu et commenté, de sorte que les correspondances de Chauveau, maintes fois repiquées et débattues dans les journaux canadiens, atteignent tout autant, comme par diffraction, le lectorat canadien. Elles vont forger la réputation de leur auteur à l'intérieur du pays et l'étendre bien au-delà de la ville de Québec. Dans les commentaires de la rédaction du Courrier, on saisit que le correspondant prend de la valeur. Le 28 septembre 1844, Gaillardet annonce la souscription en vue de la publication de l'Essai sur la littérature française depuis 1789 , en soulignant que «P. Chauveau, quoique jeune encore, s'est placé du premier coup à la tête des hommes les plus remarquables du Canada, comme écrivain, comme observateur et comme légiste ». Encore en 1852, mentionnant la parution de la quatrième livraison du roman Charles Guérin, Le Courrier des États-Unis rappellera qu'il est l'«œuvre d'un des hommes politiques les plus distingués du Canada» (18 novembre 1852). Le jeune poète romantique inquiet s'est effacé entre-temps.

littératures francophones en dialogue: du Québec et de la Suisse romande, Québec, Presses de l'Université Laval, 2004, p. 221-232. 